\title{
EDITORIAL
}

\section{WILL ANTIBIOTIC MISUSE NOW STOP?}

A recent $\mathrm{WHO}$ report has added new impetus to the argument that the use of antibiotics as growth promoters should be banned.

The use of antibiotics as growth promoters in the economically important livestock industry is a key area of conflict between public health interests and the interests of big business. Low doses of antibiotics administered to animals can increase their growth rates, presumably because subclinical infections that compromise the animals' ability to thrive are suppressed; the animals grow faster and more efficiently, with a subsequent reduction in costs and obvious economic gains for farmers. In addition to the economic benefits for agriculture, this practice is of significant importance to the antibiotic industry. It has been estimated that as much as $50 \%$ of total antibiotic production (by weight) is used in animals and plants, with $50-80 \%$ of antibiotic use in some countries devoted to growth promotant and disease prevention purposes.

The main cost of the use of antibiotics as growth promoters, however, is the development of antibiotic resistance. Many studies have shown an irrefutable link between the subtherapeutic use of antibiotics and the development of antibiotic resistance in bacteria. Given that the antibiotics used to treat livestock are often identical to medically important antibiotics used to treat humans, it would seem obvious that the development of resistance against these drugs could have an important and direct impact on public health. However, this is a controversial issue. The impact of the use of antimicrobials in agriculture on human health has been a subject of debate, discussion and risk assessments for decades and, although there is a consensus that antimicrobial use in animals selects for resistance in zoonotic pathogens and these resistant bacteria can be transmitted to humans through contact with animals or food, there is less agreement on the overall extent of the impact on public health. Why? A major barrier is the fact that many scientists involved in agriculture and food animal producers refuse to accept that the use of antibiotics in livestock has a negative effect on human health. This attitude has been strengthened by a lack of sound experimental studies documenting the impact; fortunately, a growing number of reports are now beginning to provide clarification.
In 1998, Denmark became the first country with a significant livestock industry to curtail the use of growth promoters in pig and poultry production. The World Health Organization (WHO) report analysed the effects of the ban on the prevalence of antibiotic resistant bacteria in farm animals, abattoirs and meat over a 4 -year period. The impact of the ban on animal health and the economic costs to farmers were also assessed. It was concluded that although there was a slight negative economic impact, the ban on growth promotants achieved its aims - a $54 \%$ decrease in total antibiotic use and a dramatic fall in the number of drug-resistant strains isolated from animals and meat. For example, the proportion of Enterococcus bacteria sampled from animals that were resistant to common antibiotics fell from $60-80 \%$ to $5-35 \%$. In the clinic, enterococcal infections present severe treatment problems, particularly in immunocompromised patients because these bacteria have become resistant to all available antibiotics. 'Under conditions similar to those in Denmark', the report concludes, 'the use of antimicrobials for the sole purpose of growth promotion can be discontinued'.

It is understandable that the food-producing industry wishes to protect its interests. However, microbiologists are aware of, and understand, the weight of evidence linking the subtherapeutic use of antibiotics with the emergence of resistant bacteria. Microbiologists also understand the threat that antibiotic resistance poses to public health. As a profession, we must be vocal in supporting any policy that diminishes this threat. To that end, we welcome the fact that the European Union has finalized rules to end the practice in all member countries by 2006 and that legislation has been introduced in the United States that would phase out the routine use of clinically important antibiotics by the livestock industry. As researchers, microbiologists can further contribute in two ways. First, by adding to the data linking antibiotic use in agriculture to effects on human health. Second, by using our knowledge of bacterial genomics and pathogenesis to develop new antiinfectives that are specific against pathogens of animals but do not generate resistance in pathogens of humans. 